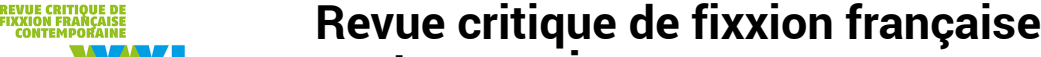 contemporaine
}

$20 \mid 2020$

Radicalités

\section{Relire Hélène Bessette, Ida ou le délire (1973)}

Dominations, aliénations, résistances, à la racine du mot

\section{Hélène Sirven}

\section{CpenEdition}

\section{Journals}

Édition électronique

URL : https://journals.openedition.org/fixxion/630

DOI : $10.4000 /$ fixxion. 630

ISSN : 2295-9106

Éditeur

Ghent University

Référence électronique

Hélène Sirven, «Relire Hélène Bessette, Ida ou le délire (1973) 》, Revue critique de fixxion française contemporaine [En ligne], 20 | 2020, mis en ligne le 15 juin 2020, consulté le 18 mai 2022. URL : http:// journals.openedition.org/fixxion/630; DOI : https://doi.org/10.4000/fixxion.630

Ce document a été généré automatiquement le 18 mai 2022

\section{(c) (†) $\ominus$}

Les contenus de la Revue critique de fixxion française contemporaine sont mis à disposition selon les termes de la licence Creative Commons Attribution - Pas d'Utilisation Commerciale - Pas de Modification 4.0 International. 


\section{Relire Hélène Bessette, Ida ou le délire (1973)}

Dominations, aliénations, résistances, à la racine du mot

Hélène Sirven

\section{Prétextes}

1 Tout au long de sa carrière d'écrivaine, Hélène Bessette (1918-2000) mena avec une énergie farouche des actions littéraires et graphiques en quelque sorte prosopoiétiques, c'est-à-dire au moyen d'une prose poétique dont le processus de création est rendu visible: on a l'impression d'être dans la tête de l'écrivaine pendant qu'elle écrit et choisit ses mots, ses effets, elle nous interpelle plus ou moins directement au cours d'un récit à plusieurs voix. Dans Ida ou le délire ${ }^{1}$ - dernier ouvrage paru de son vivant le 27 mars 1973 - elle déploie avec agilité seize morceaux choisis ${ }^{2}$ que l'on pourrait imbriquer (ou pas) pour tenter d'expliquer les causes de la mort accidentelle de l'héroïne, tout en sachant que c'est d'évidence impossible et au fond sans intérêt. Le dispositif stratégique mis en œuvre par Bessette promeut une nécessaire littérature de résistance à toute forme de domination, par l'usage de la collision, de la juxtaposition, de la répétition des mots et des idées ${ }^{3}$. Son écriture libre s'inspire des textes de Gertrude Stein (Three Lives, 1909, dont "The Good Anna"), mais aussi des films d'Ingmar Bergman ${ }^{4}$, entre autres. Ainsi, Ida ou le délire (une vie banale, un accident qui fait jaser et réactive différents souvenirs et conversations chez celles et ceux qui l'ont connue) serait un prétexte : l'auteure utilise les ressorts de cette histoire banale, s'en amuse, la déploie dans différentes directions (échanges entre divers interlocuteurs) pour nous inciter à changer notre mode de lecture. Bessette joue à sa manière avec l'anecdote, le fait divers, en déviant au fond les apports cliniques du Nouveau Roman (Alain RobbeGrillet, Pour un nouveau roman, 1963). Bessette s'écarte en effet quelque peu de cette théorie littéraire en réintroduisant une échappée poétique, parfois grinçante, dans la médiocrité ravageuse du quotidien, souvent avec humour. Parmi les auteurs qu'elle affectionnait, mentionnons Virginia Woolf (A Room of One's Own, 1929), sa fin tragique, les voix qui minaient son monde intérieur. L'histoire de la pauvre Ida, dont la chambre 
était interdite à sa patronne, Madame Besson, résonne comme dans une pièce où l'on peut enfin se retirer. Les bruits et la violence du dehors, les opinions, les jugements des autres, s'épuisent à vouloir pénétrer son lieu intérieur. La vieille servante Ida possédait sa chambre à elle. Et le roman poétique de Bessette devient une chambre à soi.

Le portrait d'Ida Schmidt, renversée par un camion, est au fond prétexte à affirmer les codes bessettiens de la révolte, l'attention aux petits de ce monde, et à tous les petits bruits des mots, le tressage entre l'écrit et l'oral au profit d'une nouvelle expérience de récit poétique, fluide, rythmée comme un morceau de jazz. Bessette mélange les langages, bouleverse la continuité de la lecture: le livre commence par la mort de l'héroïne, donc par la fin, et différentes conversations s'intercalent. Cette lecture est cependant préservée d'une autre manière grâce aux espaces blancs d'écriture dans la mise en page, à l'instar d'un songe mallarméen, d'une respiration, d'une partition décalée, avec des points d'orgue. Et puis, le surgissement des mots imprimés en capitales revient comme une vague ("ATTENTION"; "RIEN"; "NOUS PENSIONS QU'ELLE ÉTAIT COMME NOUS”; “FACE À L'ÉPOUVANTE”; “MAIS C'EST UN SUICIDE” : “NON”). La digestion des jeux surréalistes, qui ont intéressé Besette, où la folie n'est jamais loin, part maudite des dons de la rencontre, donne au corps de l'œuvre sa gravité onirique et sa nécessaire opacité. Subsiste le fil du récit, vagabond, à plusieurs voix, où la bêtise, l'incompréhension, se heurtent à l'énigme silencieuse du personnage principal fabriqué par Bessette. "Et l'erreur, comme toujours, fait le roman." Ida, c'est une erreur de la nature, trop bizarre, trop solitaire. Sa mort est une erreur, elle aurait dû faire attention en traversant la rue! Les gens de bien se sont trompés sur son compte; bref, toute une série d'erreurs finit par construire l'énigme du roman : on ne saura jamais à coup sûr si elle s'est suicidée ou pas.

La vieille Ida se disait oiseau nocturne. Elle collectionnait les chaussures, regardait trop ses grands pieds. Oui, elle aimait arroser les fleurs - bizarrement, la nuit. Gardant à jamais tout son mystère, Ida est à la fois ailleurs et virtuellement là, on parle d'elle après coup, on bavasse sur son existence passée, prétexte à affirmer le pouvoir des nantis. Les conversations s'égarent, se répètent, on divague, et les réminiscences participent de la théâtralisation du fait divers, des "vies minuscules", de l'ennui. Bessette aimait les romans policiers et s'est frottée avec un plaisir non dissimulé à ce genre littéraire (Vingt minutes de silence, 1955). L'auteure enquête, ou fait semblant d'enquêter, car elle semble nous déléguer cette tâche. Laissant ouvert le champ des possibles, elle décrit les aléas de la prise de possession de certains individus par d'autres : la vie et la mort d'Ida sont la conséquence de la domination exercée par les maîtres. Le sordide, l'aliénation et la souffrance façonnent inexorablement le personnage d'Ida. Et Bessette joue sur une forme de distance anthropologique face à l'inéluctable disparition de tout être vivant, tout en créant un simulacre d'observation participante qui prolonge les effets du texte. La littérature survit en principe à la mort de l'auteur, sauf quand l'auteur est oublié.

\section{Contextes}

4 En 1956, Hélène Bessette fonde le Gang du Roman poétique (G.R.P.) et en 1969, elle imprime cent exemplaires de son manifeste intitulé Le résumé, où elle précise son positionnement littéraire: le roman poétique est une réponse au contexte contemporain et la littérature doit rattraper son retard. Il y a d'autres façon d'écrire, de 
composer, en mettant en exergue les liens entre la parole et les mots du texte, les expériences sonores, artistiques des avant-gardes (lettristes, situationnistes, performances et la révolution de John Cage, etc.) ont libéré la manière de raconter l'histoire, ou de ne plus vraiment raconter d'histoire, au profit de la texture de la page, de son souffle. La beauté des mots, leur musicalité, leur couleur définissent des archipels de sens littéral d'où monte la vision poétique: le livre devient un objet artistique frémissant.

5 Ida ou le délire (2 200 exemplaires) possède une valeur tragique : c'est à partir de 1973 que Gallimard décide de ne plus publier Hélène Bessette. Elle a cinquante-cinq ans, s'enfonce dans la précarité, la maladie et la désolation, une mort lente est en route. De fait, son œuvre reste inachevée. Le scandale des Petites Lecocq (1955) - la bibliothécaire Jacqueline Wolfgang-Leiner, née Lecocq, se reconnaît et demande une somme exorbitante en dommages et intérêts, le procès est ruineux pour Bessette - a contribué à sa chute littéraire et personnelle. Ses rapports avec Gallimard se dégradent, on ne répond plus à ses lettres désespérées et insistantes, on ne veut plus de ses textes. "L'échange est impossible et l'on comprend peut-être mieux pourquoi la maison d'édition a attendu sept ans, après Suite suisse, pour publier Ida ou le délire." 6

Ida n'est pas tout à fait autobiographique, puisque Bessette - néanmoins femme de ménage, faute de mieux - dit s'être inspirée d'une histoire vraie, arrivée en 1971 près de Lausanne à Renens? .

7 Aujourd'hui encore, l'intransigeance d'Hélène Bessette, extrêmement active, interactive, déstabilisante, qui ne lâche rien, confine à la mise en abyme dangereuse de l'aliénation. Chez elle, la folie est l'ultime accident de l'injustice sociale, du déni de reconnaissance, de la violence. La rencontre avec l'œuvre d'Hélène Bessette permet d'aborder des formes particulières de récit et d'interpellation, une prise de conscience par les voies du roman poétique. Lire ses textes, c'est entendre une voix, sa voix, en découvrant l'inventivité de ses choix formels et de ses dispositifs d'écriture : archipels de mots, phrases cadencées, décalées, elliptiques, blancs, répétitions, suppressions ou ajouts de ponctuation, juxtapositions, etc. : "IDA / elle a été accidentée /Et lui, l'homme qui interroge a vu Ida / Comme elle devait être vue / Le cadavre-Ida / Ne parlons pas de ça. Dit-il brièvement. / Vous ne répondez pas à la question. / Madame Besson parle trop. / - Ah bien, dit-elle, si vous voulez savoir ce qu'elle était. / C'est différent"'.

Le processus créatif bessettien relève d'une pratique artistique éprouvée par son histoire personnelle et les chemins qu'elle a empruntés depuis des terres lointaines. Car Bessette commence en fait sa carrière d'écrivaine en Nouvelle-Calédonie (1946-1949). Elle est alors l'épouse du pasteur René Brabant et le couple de missionnaires dirige la revue Évangile-Sud (1947-1948). Marie Désoublie, feuilleton signé Hélène Brabant, est remarqué par le pasteur et ethnologue Maurice Leenhardt. Grâce à lui, à Michel Leiris, puis à Raymond Queneau, la maison Gallimard ouvre ses portes dès 1952 à Hélène Bessette, la divorcée, rentrée en France en catastrophe. Son biographe Julien Doussinault remarque dans ce roman-feuilleton inaugural des éléments caractéristiques de l'écriture bessettienne: "Ce premier roman schématise dans une certaine mesure le sentiment religieux en laissant apparaître quelques trouvailles ultérieures qui feront plus tard le style d'Hélène Bessette: descriptions, monologues intérieurs, drames de roman noir ou encore jeux typographiques. Il y a déjà dans Marie Désoublie la forme que l'on a découverte dans Lili pleure et Vingt minutes de silence, ainsi qu'un personnage (Ida) qui apparaîtra dans un livre publié vingt-cinq ans plus tard"9. 
La déchéance de Marie (dans l'hôtel de Madame Ida) est rachetée par sa conversion au catholicisme. Même si la spiritualité ne semble pas avoir déserté les romans poétiques de Bessette - bien revenue de son engagement religieux - la vieille bonne Ida ne se convertit à rien et échappe brutalement à ses maîtres. La réalité physique de son cadavre fixe la trace peut-être suicidaire de sa disparition. Les derniers mots du roman l'attestent : "IDA EST MORTE" au milieu de la page blanche. Comme une inscription sur une pierre tombale ou, en raison de sa formulation, comme un bandeau dans un journal local ou un cri.

9 Le franchissement des limites, mine de rien, transforme Ida ou le délire en cri radical, une insurrection, peut-être contre-fictionnelle ${ }^{10}$. Radical ? C'est-à-dire "profond, intense, total, absolu"11. Contre-fictionnel? L'irruption combattive de la réalité du roman poétique bessettien dépasse au final toute tentative de récit imaginaire, d'illusion, par l'usage d'une langue hybride (le parler et l'écrit tressés, tendus comme un arc) et une forme de "rapport" des faits. Ce rapport surgit, déborde, en un débit presque onirique, sans pour autant lâcher toutes les opportunités de la fiction: inventer des configurations toniques, jouer avec les mots du réel enfui pour activer la puissance créatrice du texte. Autrement dit, on n'imagine pas que Bessette imagine, elle suscite plutôt des images mentales tout en jetant des instants de vie apparemment bruts. Ces moments prennent racine avec une virtuosité désarmante, dans un flux scandé par les espaces blancs de la page, pleins d'imprévisible.

\section{Corps des mots, mots du corps}

10 La manière dont "fonctionne" Ida ou le délire pourrait être une version à la fois bouillonnante et glaciale d'Un cœur simple (1877) - dernière œuvre publiée du vivant de Gustave Flaubert. La description rigoureuse et terrible de l'histoire de la pauvre Félicité résonne dans celle de l'énigmatique Ida. Toutes deux gardent leur secret.

11 Ida, c'est un corps, informe, difforme, un tas de vêtements après l'accident ; le roman poétique bessettien, organisme rhizomique, fait corps avec son auteure, et la partition d'Ida participe d'un vaste corpus, (15 romans, une pièce de théâtre), d'un langage élaboré au cours du cheminement chaotique, déterminé, d'Hélène Bessette - femme, mère, institutrice, un temps enseignante, puis missionnaire, et surtout écrivaine envers et contre tout.

Que dit Ida des filiations, des résiliences, et de quelle dérive s'agit-il ? Comment s'insurge-t-elle dans cette langue picturale, musicale, errante ? Il y a en effet dans l'œuvre exploratoire bessettienne, y compris dans son théâtre, une pratique de l'écriture en prose expérimentale (musicale, cinématographique et picturale), des interjections en lien avec les avant-gardes artistiques, une porosité à l'esprit du temps si bien décrit par Edgar Morin -, en rupture avec un récit continu, "réaliste". Et les silences, les ponctuations, la mise en page aussi, la typographie, l'usage des adjectifs, des répétitions, des récurrences, d'un langage familier, toujours direct, participent de son geste littéraire. Une adresse au lecteur fulgurante et soigneusement construite le fait dériver vers de nouvelles situations d'exil volontaire. Bessette s'est définie comme une exilée, elle nous emmène dans cet exil intérieur et voyageur.

13 Ida ou le délire ressemble à un procès, extime et intime, qui condamne la société des maitres et des serviteurs, des esclaves ${ }^{12}$ en éclairant le destin des humbles, des taiseux, des opprimés, des femmes reléguées à une place subalterne. Comment la question de 
l'estime de soi, de la dignité, apparaissent-elle dans Ida? Par défaut. Une critique acerbe, politique, du monde du travail, de la famille bourgeoise, de l'indifférence sociale, est sous-tendue par la description de faits, assortie d'un humour-ferment, par des mots, des images, des commentaires ("Elle était très intelligente / Trrrrès intelligente / Madame Besson crie / Affirme / Affirme en criant. Crie en affirmant" ${ }^{13}$ ). Le suicide présumé, en tout cas la mort brutale d'Ida, réponse radicale, dans toutes les dimensions de l'abandon, nous concernent toujours. Hélène Bessette semble croire en la littérature, mais une littérature en mouvement dont elle redéfinit le langage et la méthode. Elle veut transmettre la nécessité d'écrire, sans répit intérieur, en maintenant le secret et l'oxymore de ce secret : ne pas dire tout en disant. Elle apporte ainsi sa connaissance de la douleur, du désir, du rejet, et son obstination. Le théâtre de la vie qu'elle scrute devient alors un réquisitoire et un appel. Les mots d'un corps indissocié de l'esprit s'enracinent dans la protestation.

Comme dans les prières évangélistes qu'elle a dites, les psaumes, comme dans les souffles épiques des ténô kanak ${ }^{14}$ qu'elle a peut-être écoutés là-bas, Bessette utilise "le présent permanent". Sur un mode elliptique, sans virgules, parfois sans point, ces phrases scandées expriment la force du moment de l'énonciation: "Dans la nuit de cauchemars / Avec somnifère / Autour de Ida / Ces peuples de Reines / Drapées d'or, ruisselant de diamants et de pierres. Ces carrosses ces peintures ces meubles ces bronzes ces marqueteries. / Ces Princes en velours dentelles soieries / Pour emplir le Délire de Ida / Ida Délirante / aux milliers d'images historiques / aux grandeurs affolantes / hallucinantes. / Peut-être intelligente. / Oui. Peut-être / Ce peu d'intelligence / Ce très peu d'intelligence / comme le soleil dissimulé derrière la montagne. Et son / reflet blanc visible sur le paysage glacé" ${ }^{15}$. Cet exemple, parmi tant d'autres dans Ida, illustre l'analyse de Doussinault de la détermination bessettienne, où l'on reprend sans cesse le fil de l'histoire : “Coïncider avec ce que l'on pense, ignorer le passé, s'affranchir de la mémoire, des douleurs du temps ; pour mieux saisir la vérité du moment, c'est aussi ce que s'efforcera de faire Bessette par le moyen de la 'reprise' qui, dans Ida ou le délire par exemple, figure la présence permanente de l'instant, 'ce point de durée sans durée"”'16.

\section{Ouvrir la littérature}

La servante Ida meurt projetée par un camion, dans une élévation grotesque, fulgurante et spectaculaire. Autre cœur simple, la flaubertienne Félicité crut voir apparaître au moment du grand passage un immense perroquet, planant au-dessus d'elle, dans les cieux entrouverts. En somme, la suspension définitive est le contrepoint de la chute, ultime oxymore désolant qui relie les cicatrices de la basse condition abîmée à une forme d'extase aérienne délirante. L'ekphrasis se disloque en images dispersées, à l'instar d'un cadavre exquis, tout en exaltant la virulence des mots. La description de l'accident mortel d'Ida relève aussi du montage cinématographique ; on la voit projetée dans les airs. Différemment, les dernières lignes qui narrent la mort de Félicité fixent l'apparition étrange de l'oiseau en gloire, éventuellement psychopompe, et on croit voir cette monstruosité sacrée. L'histoire dans ces deux situations relève de la confusion des genres, du fait divers transcendé, où le banal rendu extraordinaire par l'acte littéraire révèle la complexité discontinue de l'ordre des choses. C'est dans le désordre assumé, aléatoire, cultivé paradoxalement en friches de désir et lieux de 
création que l'écriture peut développer ses espaces temporels. Bessette, semble-t-il, y a laissé l'empreinte récurrente et douloureuse, absolue, de sa destinée, en nuages de mots colorés, cinglants, drôles.

Hélène Bessette a voulu ouvrir le corps de la littérature, pour plonger dans ses abymes. Son testament poétique, à l'épreuve des faits, peut-être encore aux marges de l'histoire, manifeste une intense vitalité. Le Colloque de Cerisy, en 2018, célébration du centenaire de la naissance de l'écrivaine, avec ses prolongements éditoriaux, théâtraux, artistiques et littéraires ${ }^{17}$, a mis en urgence l'injonction de Marguerite Duras dans L'express de 1964 : "Lisez Hélène Bessette".

\section{NOTES DE FIN}

1. Hélène Bessette, Ida ou le délire, Paris, Othello, Le Nouvel Attila, 2018.

2. Ou "tableau", selon Julien Doussinault, dont l'excellente et seule biographie à ce jour d'Hélène Bessette est la référence principale de mon article : Hélène Bessette. Biographie, Paris, Éditions Léo Scheer, 2008. Et à propos d'Ida ou le délire, voir en particulier p. 247-271.

3. "On voit les gens aller et venir. Puis on découvre qu'ils sont des malades bons pour les cliniques. Ils travaillent ils mangent ils parlent. Puis on découvre qu'ils sont des pourvoyeurs d'hôpitaux. Avec des somnifères la nuit. Comprimés anti-fièvre le jour. Avec eczéma. / Elle avait une pommade et enveloppait ses bras et ses mains chaque soir. / On les voit aller et venir. Puis on apprend qu'ils tiennent (debout) à coups de bandelettes de bandages de pansements de produits pharmaceutiques. / Des représentants en pharmacie. / Sans sommeil. Avec fièvre. Avec eczéma. Avec calmants. / Contagieux. Avec soins. Avec vaseline. Avec tubes et petits pots. / Des laboratoires ambulants. / Des sujets d'expérience en promenade dans les rues de la ville." Hélène Bessette, op. cit., p. 78.

4. "Dans Persona par exemple, certaines scènes se répètent, des insertions quasi subliminales apparaissent sur la pellicule et l'image, tantôt "floutée", tantôt accélérée, constamment mise à l'épreuve, finit même par brûler sur l'écran. Les rapprochements entre les livres d'Hélène Bessette et les films de Bergman sont plus flagrants encore quand ils concernent les thèmes abordés, en accord le plus souvent avec une philosophie particulièrement pessimiste, largement inspirée de Kierkegaard et de Schopenhauer." Julien Doussinault, op. cit., p. 263.

5. Hélène Besette, op. cit., p. 11.

6. Julien Doussinault, op. cit., p. 263.

7. Ibid., p. 247.

8. Hélène Bessette, op. cit., p. 93.

9. Julien Doussinault, op. cit., p. 66.

10. Yves Citton, “Contre-fiction : trois modes de combat”, Multitudes, $\mathrm{n}^{\circ} 48,2012$, p. 72-78.

11. De radicalis, "qui tient à la racine, premier, fondamental" et de l'anglais radical, "absolu, complet", (CNRTL en ligne).

12. "Son enfance lointaine. Soixante ans en arrière. Estompée dans le brouillard des mémoires entassées. Son enfance? Sans enfance. Ida adulte et responsable. Les yeux clos sur une énorme responsabilité. Celle des mondes enchaînés de l'esclavage. Et cette voix bienveillante (pour débile et déficient) ne lui convient pas. Il faut le dire. Ida ne supporte pas la bienveillance. Alors elle se 
tait. Tout au plus elle rit. Pour masquer la colère montante. La grande colère. Et ce n'est pas la moindre des colères." (Hélène Bessette, op. cit., p. 26).

13. Ibid., p. 91.

14. Alban Bensa, Kacué Yvon Goromoedo, Adrian Muckle, Les sanglots de l'aigle pêcheur. Nouvelle Calédonie : la Guerre kanak de 1917, Toulouse, Anarcharsis Éditions, 2015, p. 433-679. "Le rythme du ténô rompt avec la parole ordinaire et constitue l'expérience en évènement, tout en favorisant la mémorisation, c'est-à-dire la répétition du souvenir. La scansion joue dans la parole énoncée un rôle essentiel. Appuyé sur une versification de huit pieds et toujours dit à grande vitesse, le ténô dégage un effet de litanie et de souffle qui, au-delà d'un sens littéral, porte une exaltation épique proche du chant." (p. 615)

15. Hélène Bessette, op. cit., p. 98.

16. Julien Doussinault, op. cit., p. 145.

17. Le colloque qui s'est tenu à Cerisy-la-Salle, "Hélène Bessette : l'attentat poétique" (20-27 août 2018), organisé par Julien Doussinault, Claudine Hunault et Cédric Jullion, a rendu hommage à Hélène Bessette, en présence de ses fils Éric et Patrick Brabant. Le GAP reconstitué continue ses actions, avec l'engagement littéraire des Éditions du Nouvel Attila, pour faire connaître l'œuvre d'HB7 sous différentes formes artistiques : théâtrales, performatives. Sans oublier les lectures. Léo Scheer fut le premier éditeur, avec la contribution de Laure Limongi, de 2006 à 2012, à faire redécouvrir l'œuvre de Bessette, saluée en son temps par les plus grands écrivains et artistes (Queneau, Leiris, Duras, Beauvoir, Sarraute, Dubuffet...) avant de tomber dans un triste oubli.

\section{AUTEURS}

\section{HÉLÈNE SIRVEN}

Université Paris 1 Panthéon-Sorbonne 